LAWRENCE LIVERMORE N A T IO N A L LABORATORY

\section{Comparison of Three Afterglow Morphologies}

J. D. Salmonson, E. Rossi, D. Lazzati

December 24, 2003

2003 Gamma-ray Burst Symposium

Santa Fe, NM, United States

September 7, 2003 through September 12, 2003 
This document was prepared as an account of work sponsored by an agency of the United States Government. Neither the United States Government nor the University of California nor any of their employees, makes any warranty, express or implied, or assumes any legal liability or responsibility for the accuracy, completeness, or usefulness of any information, apparatus, product, or process disclosed, or represents that its use would not infringe privately owned rights. Reference herein to any specific commercial product, process, or service by trade name, trademark, manufacturer, or otherwise, does not necessarily constitute or imply its endorsement, recommendation, or favoring by the United States Government or the University of California. The views and opinions of authors expressed herein do not necessarily state or reflect those of the United States Government or the University of California, and shall not be used for advertising or product endorsement purposes. 


\title{
Comparison of Three Afterglow Morphologies
}

\author{
Jay D. Salmonson*, Elena Rossi ${ }^{\dagger}$ and Davide Lazzati ${ }^{\dagger}$ \\ ${ }^{*}$ Lawrence Livermore National Laboratory, Liveremore, CA 94551 \\ ${ }^{\dagger}$ Institute of Astronomy, University of Cambridge, Madingley Road CB3 OHA, England
}

\begin{abstract}
.
Herein we compare three functional families for afterglow morphologies: the homogeneous afterglow with constant shock surface energy density, the structured afterglow for which the energy density decays as a power-law as a function of viewer angle, and the gaussian afterglow which has an exponential decay of energy density with viewer angle. We simulate observed lightcurves and polarization curves for each as seen from a variety of observer vantage points. We find that the homogeneous jet is likely inconsistent with observations and suggest that the future debate on the structure of afterglow jets will be between the other two candidates.
\end{abstract}

The structure of gamma-ray burst afterglows is currently a question of considerable interest. Observations of afterglows have become sufficiently numerous and well sampled that inferences can be made about the morphology and evolution of the external shock, which is thought to generate the afterglow's emission. Specifically, power-law decay slopes, jet-break times and polarization information all can be used to model the structure of the afterglow.

Several clues can be used to infer the structure of afterglow jets. A first clue to the structure of afterglows was the realization that the inferred jet opening angle is inversely proportional to the energy density in a manner suggestive that, in sum, most bursts have similar energies [1]. This realization prompted the postulate that the jet might be structured [2] and that by varying the afterglow external shock energy per solid angle [3] one might encapsulate all observations into a single quasi-universal morphology. Another clue is polarization $[4,5]$. With the measurement of polarization in a handful of real afterglows comes the possibility of using this powerful diagnositic as a tool to infer the structure of afterglows [6].

In this paper we model three basic afterglow morphologies in stark simplicity so to compare and discriminate their basic observational differences. All of these models have been previously examined in whole or in part $[3,7,8,9,6]$, but here we make a comparison of perspective effects on both the lightcurve and polarization curve for each of them. To do this we use a simple spectral model in which peak and cooling frequencies are below the optical band [3,7] and we neglect lateral expansion.

- Homogeneous Afterglow The canonical afterglow, this jet is characterized by a constant afterglow shock surface energy density, $\varepsilon$, within an opening angle, $\theta_{0}$, and a sharp, Heaviside drop-off at this edge, $\varepsilon(\theta) \propto H\left(\theta-\theta_{0}\right)$. As can be seen in Figure 1, this afterglow exhibits a viewing angle dependent two-stage break in the lightcurve whereby the flux deficit imposed by the near side of the jet coming into 


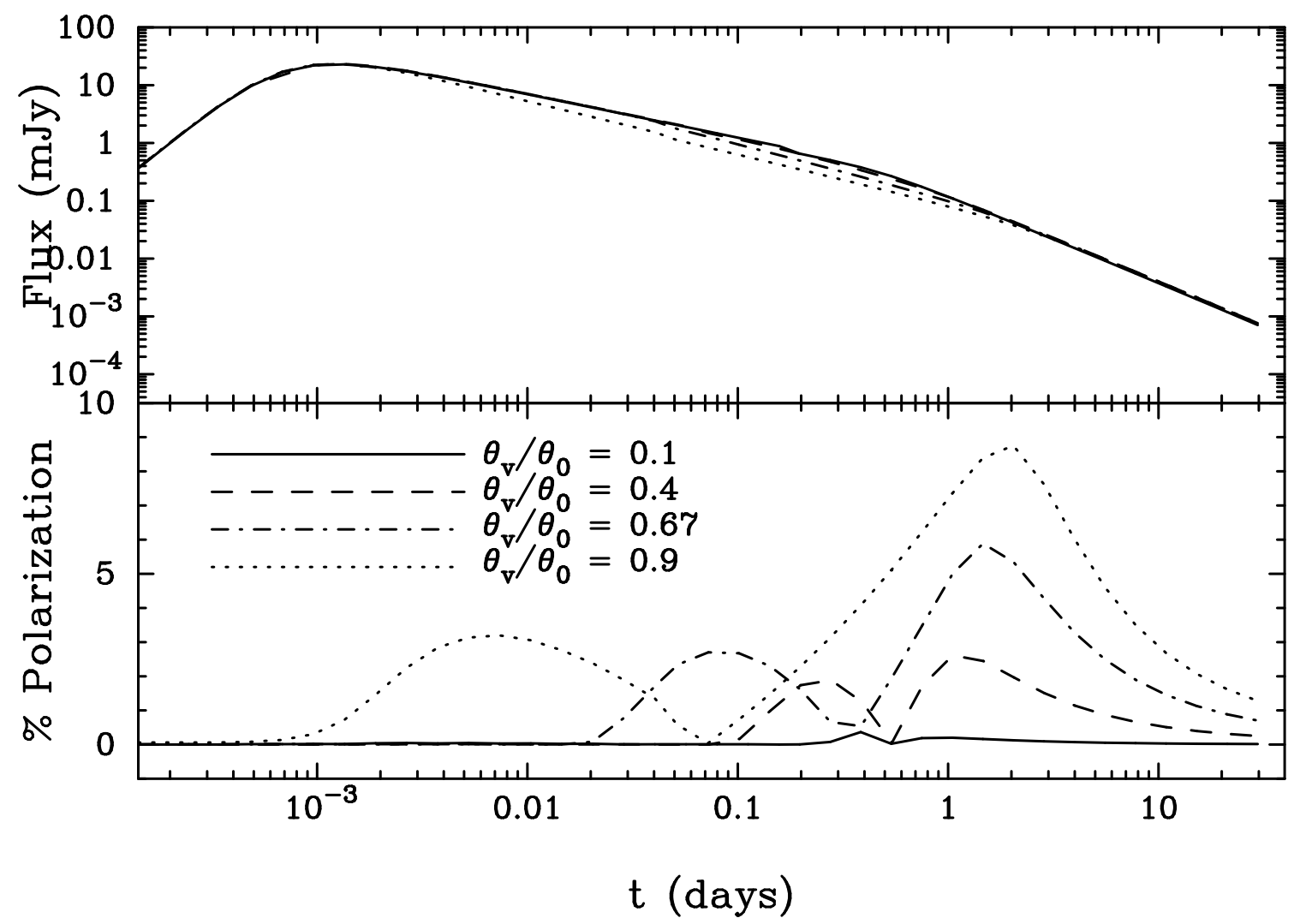

FIGURE 1. Homogeneous jet with $\theta_{0}=5^{\circ}$ as seen from a range of vantage points, $\theta_{\mathrm{v}}$. The double humped polarization curves indicate a $90^{\circ}$ shift in picth angle. Thus this modle predicts a null polarization near the jet-breakt time.

view prompts a first steepening, while a second steepening happens when the far side of the jet comes into view [7]. These two stages roughly correspond to two separate maxima in polarization separated by a $90^{\circ}$ shift in the pitch angle. Thus this model predicts roughly zero polarization at the jet break time, with orthogonal polarization directions before and after the break. To date convincing observations of such behavior have not been reported [6].

- Structured Afterglow If the afterglow shock surface energy density is a powerlaw for angles greater than some narrow core, $\theta_{\mathrm{c}}$, e.g. $\varepsilon(\theta) \propto\left(1+\left(\theta / \theta_{\mathrm{c}}\right)^{2}\right)^{-1}$, then the observed lightcurve varies significantly as a function of viewer angle. This raises the intriguing possibility of universality; that all gamma-ray bursts are created roughly equal, but the wide observed variety stems merely from the range of viewer vantage points [3]. As seen in Figure 2, a relatively wide variety of viewing angles produces a variety of lightcurve flux levels and jet-break times. Also, each viewer should see a maximum in polarization coinciding with the jet-break time. For the largest viewing angles, simulations show a flattening in the slope of the lightcurve prior to the jet-break [7]. This is not observed in the data and so provides clues and constraints on universality and structured jets.

- Gaussian Afterglow Characterized by an exponential decay of the shock surface 


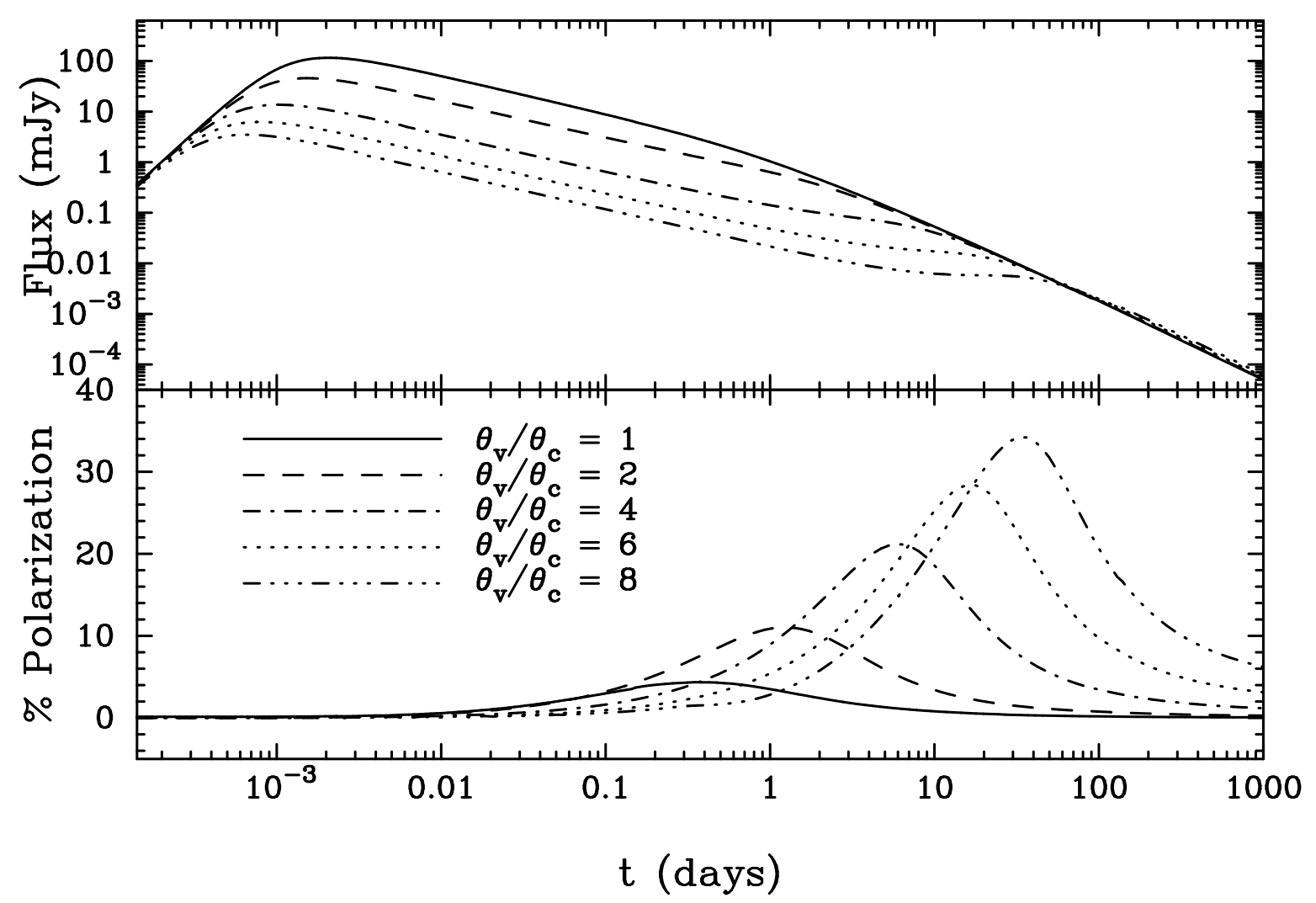

FIGURE 2. Structured jet with core angle $\theta_{\mathrm{c}}=2^{\circ}$ as seen from a range of vantage points, $\theta_{\mathrm{v}}$.

energy density, $\varepsilon \propto \exp \left(-\left(\theta / \theta_{0}\right)^{2}\right)$, this morphology might be thought of as a more physically realistic version of the homogeneous jet, with blurred edges. The differences between this model and the structured jet are more subtle. From each one can expect a maximum polarization at the jet-break time and at a constant pitch angle (Figure 3).

\section{Discussion}

The main point to be made here is that the homogeneous and structured jets are the most distinct from each other, having both divergent lightcurves and polarization curves, while the gausian jet is middling and shares key features from each. In particular, the gaussian jet lightcurve, being relatively constant as a function of viewer angle, is more like the homogeneous jet. Thus, the gaussian jet does not lend itself to a universal interpretation. Conversely, the gaussian jet has a single peaked polarization curve which peaks at the jet-break time similar to the structured jet. This simple breakdown of model behaviors will aid in the job of discriminating between this trichotomy of afterglow morphological families.

The simplistic homogeneous jet, with a predicted $90^{\circ}$ shift in polarization pitch angle at the jet-break time, is likely inconsistent with observations. We suggest that the future 


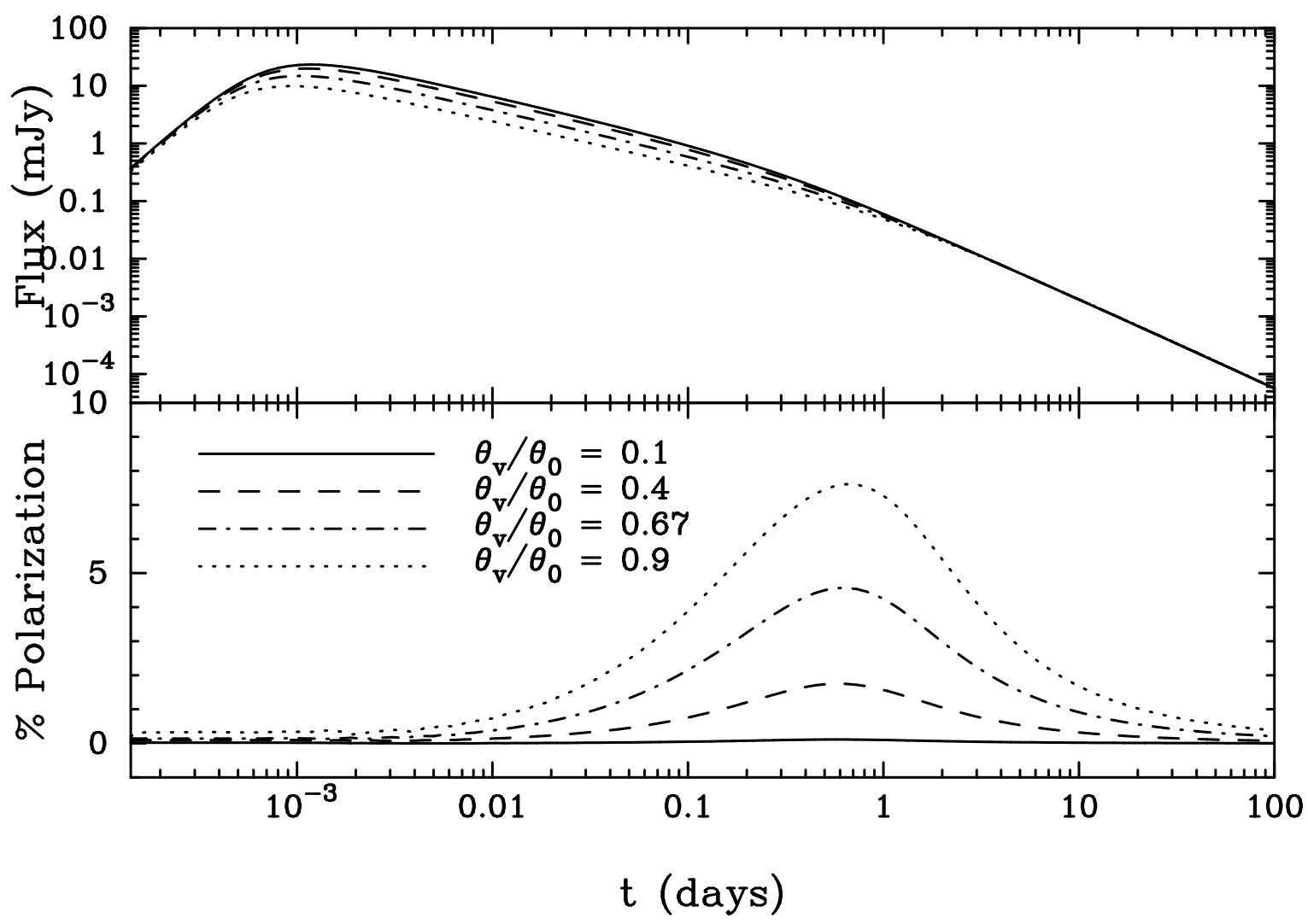

FIGURE 3. Gaussian jet with $\theta_{0}=5^{\circ}$ as seen from a range of vantage points, $\theta_{\mathrm{v}}$.

debate on the structure of afterglow jets will be between the remaining morphological candidates, each with sharp jet breaks and very similar polarization curves. The critical discriminator between these two contenders is that the structured jet lends itself to a universal gamma-ray burst paradigm while the gaussian jet requires an innate variety in the progenitor population.

This work was performed under the auspices of the U.S. Department of Energy by University of California Lawrence Livermore National Laboratory under contract W7405-ENG-48.

\section{REFERENCES}

1. Frail, D. A. et al., ApJL, 562, L55-L58 (2001).

2. Salmonson, J. D., and Galama, T. J., ApJ, 569, 682-688 (2002).

3. Rossi, E., Lazzati, D., and Rees, M. J., MNRAS, 332, 945-950 (2002).

4. Ghisellini, G., and Lazzati, D., MNRAS, 309, L7-L11 (1999).

5. Sari, R., ApJL, 524, L43-L46 (1999).

6. Rossi, E., Lazzati, D., Salmonson, J. D., Ghisellini, G., submitted MNRAS 2003.

7. Salmonson, J. D., ApJ, 592, 1002-1017 (2003).

8. Kumar, P., and Granot, J., ApJ, 591, 1075-1085 (2003).

9. Granot, J., and Kumar, P., ApJ, 591, 1086-1096 (2003). 